\title{
Effect of metallo- $\beta$-lactamase production and multidrug resistance on clinical outcomes in patients with Pseudomonas aeruginosa bloodstream infection: a retrospective cohort study
}

Matthias Willmann ${ }^{1 *}$, Ines Kuebart ${ }^{1}$, Matthias Marschal ${ }^{1}$, Klaus Schröppel ${ }^{1}$, Wichard Vogel ${ }^{2}$, Ingo Flesch ${ }^{3}$, Uwe Markert ${ }^{4}$, Ingo B Autenrieth ${ }^{1}$, Florian Hölz| ${ }^{1}$ and Silke Peter ${ }^{1}$

\begin{abstract}
Background: Blood stream infections (BSI) with Pseudomonas aeruginosa lead to poor clinical outcomes. The worldwide emergence and spread of metallo- $\beta$-lactamase (MBL) producing, often multidrug-resistant organisms may further aggravate this problem. Our study aimed to investigate the effect of MBL-producing $P$. aeruginosa (MBL-PA) and various other resistance phenotypes on clinical outcomes.

Methods: A retrospective cohort study was conducted in three German hospitals. Medical files from 2006 until 2012 were studied, and a number of 113 patients with P. aeruginosa BSI were included. The presence of VIM, IMP and NDM genes was detected using molecular techniques. Genetic relatedness was assessed through multilocus sequence typing (MLST). The effect of resistance patterns or MBL production on clinical outcomes was investigated by using multivariate Cox regression models.
\end{abstract}

Results: In-hospital mortality was significantly higher in patients with MBL-PA and multidrug-resistant $P$. aeruginosa. However, neither BSI with MBL-PA nor BSI with various resistance phenotypes of $P$. aeruginosa were independently associated with mortality or length of hospital stay. In multivariate models, the SAPS II score (HR 1.046), appropriate definitive treatment (HR range 0.25-0.26), and cardiovascular disease (HR range 0.44-0.46) were independent predictors of mortality. Concomitant infections were associated with an excess length of stay $(H R<1)$.

Conclusions: Medication with appropriate antimicrobial agents at any time during the course of infection remains the key for improving clinical outcomes in patients with P. aeruginosa BSI and should be combined with a strict implementation of routine infection control measures.

Keywords: Bacteremia, Antimicrobial therapy, Mortality, Prognosis, Length of stay, MBL

\footnotetext{
*Correspondence: will80@gmx.de

'Institute of Medical Microbiology and Hygiene, University of Tübingen,

Elfriede-Aulhorn-Str. 6, 72076 Tübingen, Germany

Full list of author information is available at the end of the article
} 


\section{Background}

Pseudomonas aeruginosa is an important nosocomial pathogen [1]. Blood stream infections, primarily observed in immunosuppressed individuals, are associated with high mortality [2,3]. The worldwide emergence of multidrug-resistant $P$. aeruginosa over the course of the last decades called the reliance on various antimicrobial agents into question [4-8]. The production of metallo- $\beta$ lactamase (MBLs) which confer resistance to all $\beta$-lactams except aztreonam is a mechanism of increasing clinical importance, largely driven by the international spread of MBL-producing organisms [9]. Therapeutic options against such strains are often severely compromised since other determinants of resistance tend to be linked to MBL genes on the same plasmid or integron structure [10,11]. Moreover, MBLs cannot be inhibited by currently approved $\beta$-lactamase inhibitors [12]. Several types of MBLs have been described, most importantly the IMP-type, VIM-type, and NDM-type enzymes [9].

Despite the growing body of epidemiological reports of metallo- $\beta$-lactamase-producing $P$. aeruginosa (MBLPA) $[8,9,13]$, more information about the clinical characteristics of MBL-PA infections is urgently needed. Most notably, the association between MBL-PA infection and clinical outcomes remains an unresolved issue. In two studies, a higher frequency of infection and fatal cases in patients with MBL-PA has been described $[14,15]$. Zavascki et al. also reported on a higher mortality among patients with MBL-PA nosocomial infections, but concluded that this result is most likely due to a delayed administration of appropriate treatment and the severity of the infections [16].

The aim of this study was to investigate the impact of MBL-production and different phenotypes of resistance on mortality and length of hospital stay in patients with $P$. aeruginosa blood stream infection and to shed some light on possible additional factors influencing such a relationship.

\section{Methods}

\section{Setting and patients}

The retrospective cohort study was performed from $1^{\text {st }}$ January 2006 until $31^{\text {st }}$ January 2012 in a 1500-bed tertiary teaching hospital, a 300-bed trauma center and a 500-bed district hospital in Tübingen, Germany, and the surrounding community. A broad spectrum of medical services is provided by these hospitals, including various surgical and medical specialties, pediatric units, dialysis and a maternity ward. Organ transplantations are performed at the tertiary teaching hospital. The study is reported pursuant to the STrengthening the Reporting of OBservational studies in Epidemiology (STROBE) guidelines [17]. The study has been approved by the local research ethics committee of the University of Tübingen (reference number: 035/2012R).

\section{Study design and definitions}

Adult patients ( $\geq 18$ years) suffering from a blood stream infection (BSI) with $\geq 1$ blood culture positive for $P$. aeruginosa were considered eligible. Patients were excluded if they were not admitted to the hospital. Hybase software (Tieto GmbH, Eschborn, Germany) was used to retrospectively identify eligible patients from the laboratory information system. Every patient was included only once at the time of the first positive blood culture (index culture).

In-hospital mortality for any cause and length of hospital stay were the primary outcomes, while exposure to strains of $P$. aeruginosa producing an MBL enzyme or to isolates exhibiting different resistance phenotypes was the variable of interest. Multidrug-resistant $P$. aeruginosa (3/4MDR-PA) was defined as resistant to at least three of the following antimicrobial agents: piperacillin-tazobactam; ceftazidime; meropenem; and ciprofloxacin [18]. For the definition of multidrug resistance we considered antimicrobial agents which have a bactericidal effect on $P$. aeruginosa and can be administered as effective monotherapy. Thus, aminoglycosides were not a part of the definition [18]. Intermediately susceptible isolates were deemed resistant. MBL-PA was defined as MBL-producing organism regardless of the susceptibility pattern.

Patient files were reviewed by medically trained personnel. Clinical data obtained included age; sex; site of infection (primary, secondary, vascular catheterrelated) according to the definition of the International Sepsis Forum [19]; nosocomial infection (defined as infection that occurred $\geq 48$ hours after hospital admission); baseline diseases; Charlson comorbidity score at admission [20]; immunosuppression, such as HIV and/or neutropenia (neutrophil count $\leq 1000 \mathrm{cell} / \mu \mathrm{l}$ ) and/or immunosuppressive chemotherapy within the previous two months (anti-cancer drugs and anti-inflammatory monoclonal antibodies) and/or receipt of steroids (prednisolone $\geq 10 \mathrm{mg}$ /daily or equivalent dose); previous surgery during hospitalization; and the presence of concomitant infections with relevant pathogenic organisms other than $P$. aeruginosa. The individual physiological condition was assessed by determining the simplified acute physiology score II (SAPS II) of the index culture day [21]. If a SAPS II parameter was not available for the index culture day, it was gained from the time point closest to the index culture day ( \pm 48 hours).

Appropriate antimicrobial treatment was defined as systemic administration of at least one antimicrobial agent to which the isolate was in vitro susceptible. Monotherapy with aminoglycosides was not considered appropriate despite in vitro susceptibility. Appropriate antimicrobial treatment was categorized in i) appropriate empirical treatment (AET) which was administered within 24 hours after samples for blood cultures were drawn, and ii) appropriate 
definitive treatment (ADT) which was administered at any time after the index culture.

\section{Species identification and phenotypic testing}

Species identification was performed through a linear MALDI-TOF mass spectrometer (AXIMA Assurance, bioMérieux, Marcy l'Etoile, France, Saramis Database Version 4.09), supplemented by Vitek 2 system identification (bioMérieux, Marcy l'Etoile, France). In vitro bacterial susceptibility testing of $P$. aeruginosa isolates was performed by use of disk diffusion tests following the EUCAST guidelines [22-24]. Colistin susceptibility was interpreted according to the CLSI breakpoints [25]. For MBL detection, a combined disk test with EDTA was performed on all isolates as previously described [26]. Briefly, antibiotic disks (Becton Dickinson $\mathrm{GmbH}$, Heidelberg, Germany) containing $10 \mu \mathrm{g}$ meropenem alone and in combination with $930 \mu \mathrm{g}$ EDTA were used. An increase of $\geq 7 \mathrm{~mm}$ in zone diameter in the presence of EDTA compared to the meropenem disk alone was considered an indication of possible MBL production.

\section{PCR assays and DNA sequencing}

$P$. aeruginosa isolates having a reduced meropenem zone size (defined as $<24 \mathrm{~mm}$ ) and/or being positive in the EDTA combined disk test were further investigated for the presence of MBL genes [26]. Whole cell DNA was used as a template in PCR assays. For the simultaneous detection of $b l a_{\mathrm{VIM}}$ and $b l a_{\mathrm{IMP}}$ genes, a multiplex PCR amplification was performed according to a protocol described elsewhere [26]. The entire VIM and IMP genes were sequenced using the primer pairs IMP-A-IMP-B or VIM2004A-VIM2004B in combination with the class 1 integron primer pair 5CS and 3CS or alternatively VIM-2SQR [26,27]. Isolates negative for VIM and IMP genes that exhibited reduced meropenem and ceftazidime zone sizes (defined as $<24 \mathrm{~mm}$ and $<16 \mathrm{~mm}$, respectively) were further investigated for the presence of NDM and class A carbapenemase (KPC and GES) genes at the National Reference Laboratory for Multidrugresistant Gram-negative Bacteria (Bochum, Germany) by using molecular detection techniques.

\section{Multilocus sequence typing (MLST)}

MLST was performed on the 3/4MDR-PA including all MBL-PA in accord with the instructions on the P. aeruginosa MLST web site (http://pubmlst.org/paeruginosa/). Internal fragments of seven housekeeping genes (acs $A$, aro $E$, guaA, mutL, nuоD, ppsA and trpE) were amplified and subsequently sequenced to determine the sequence type.

\section{Statistical analysis}

D'Agostino's K-squared test was used to check continuous variables for normality. The Student t-test was used for comparison when data were normally distributed. The Wilcoxon rank sum test was performed when transformation of variables could not achieve a normal distribution of values. A chi-squared test or-when appropriate-the Fisher's exact test were employed to compare differences in proportions.

Cox regression was used to model the relationship between the exposure (blood stream infection with MBLPA or a certain resistance type of $P$. aeruginosa) and the two outcomes (in-hospital mortality and length of stay) by calculating the hazard ratio for death and the hazard ratio for discharge (dead or alive). Exposed patients were compared with the rest of the cohort. To determine the effect on morbidity, the length of stay (LOS) was considered as failure variable. A hazard ratio of discharge $<1$ indicates a smaller hazard of being discharged and therefore an excess of stay adjusted for the length of stay before the index day (time-adjusted model) and additional confounders (fully-adjusted model) [28]. Hypothesis testing was performed by using the likelihood ratio test. Model identification for multivariate analysis was accomplished as described elsewhere [29]. Briefly, any variable with a $\mathrm{P}$-value of $<0.2$ in univariate analysis was incorporated in multivariate models, wherein only variables with a P-value of $\leq 0.1$ were retained. Excluded variables were subsequently tested for confounding by adding them one at a time to the identified model. In case of substantial confounding (defined as a change in the models' coefficients greater than 10\%) they were included into the final model. The exposure of interest was always retained in the model, regardless of the pvalue. Potential interactions were examined through the likelihood ratio test. The proportional hazards assumption was verified on the basis of Schoenfeld residuals.

All analyses were carried out by using Stata version 12.0 (Stat Corp., College Station, TX, USA). A P-value < 0.05 (two-sided) was deemed significant.

\section{Results}

\section{MBL detection and susceptibility profiles}

Over the course of the six year study a total of 6064 patients with bacterial BSI were identified. Of these, 120 patients (2\%) had a PA-BSI. Three patients were excluded because they were not admitted to the hospital. Of the remaining 117 study entrants, four patients were excluded from the analysis because clinically relevant data were missing. The remaining 113 patients were analyzed.

A number of 34 isolates met the criteria for a possible MBL production and were subsequently tested for the presence of MBL genes. Eighteen isolates (15.9\%) that possessed a gene for a metallo- $\beta$-lactamase were detected. Four isolates were positive for VIM-2 (3.5\%) and 14 isolates for IMP-8 (12.4\%). The results of the susceptibility 
Table 1 Susceptibility profile of the 113 Pseudomonas aeruginosa clinical isolates

\begin{tabular}{lll}
\hline Antimicrobial agent & $\begin{array}{l}\text { MBL }(\mathbf{n = 1 8 )} \\
\text { susceptible } \\
\text { isolates (\%) }\end{array}$ & $\begin{array}{l}\text { Non-MBL }(\mathbf{n}=\mathbf{9 5}), \\
\text { susceptible } \\
\text { isolates (\%) }\end{array}$ \\
\hline Meropenem & 0 & 85 \\
Piperacillin-Tazobactam & 0 & 83 \\
Ciprofloxacin & 0 & 78 \\
Ceftazidime & 0 & 95 \\
Colistin & 100 & 100 \\
Amikacin & 0 & 96 \\
Fosfomycin & 0 & 40 \\
\hline
\end{tabular}

MBL, metallo- $\beta$-lactamase producer.

testing are shown in Table 1. All MBL-PA were susceptible to colistin but resistant to all other tested antimicrobials. Non-MBL-PA remained susceptible to a number of antimicrobial agents, particularly to ceftazidime (95\%), amikacin (96\%) and meropenem (85\%). All P. aeruginosa isolates were susceptible to colistin.

\section{Study population characteristics}

Table 2 presents the basic characteristics of the study cohort. The median age was 64 years (IQR, 53-74 years) with $48 \%$ over 65 years. Male gender was predominant (63\%). Frequent comorbidities were cardiovascular diseases (58\%), diabetes (33\%) and hematological cancer (32\%). The most common sources of infection were the respiratory and urinary tract (19\% and $16 \%$, respectively). The source of infection was not identifiable in $44 \%$ of cases. The mean time to effective treatment was 1.05 days in our cohort.

The in-hospital mortality was $38 \%$. Differences in baseline characteristics between the two exposure groups MBL-PA and 3/4MDR-PA are shown in Table 3. Patients infected with a MBL-PA had a higher mortality than those with a Non-MBL-PA (61\% vs. $34 \%, P=0.03$ ). A BSI with a $3 / 4 M D R-P A$ resulted in a higher mortality as well $(63 \%$ vs. $30 \%, P=0.002)$. Interestingly, while patients with MBL-PA BSI overall had a higher SAPSII score (39.5 vs. $32.0, P=0.002$ ), there was merely a tendency towards a difference in the administration of definitive appropriate treatment in comparison to the Non-MBL-PA group. The opposite seemed to be true for patients with 3/4MDR-PA BSI: No significant difference in SAPSII was observed, but the number receiving appropriate definitive treatment was significantly lower $(70 \%$ vs. $90 \%, P=0.03)$. Patients infected with MBL-PA or 3/4MDR-PA were more frequently neutropenic $(\mathrm{p}<0.001)$

\section{Clinical outcomes}

Table 4 shows the results of the univariate analysis. While BSIs with 3/4MDR-PA and $P$. aeruginosa strains
Table 2 Basic characteristics of 113 adult patients with Pseudomonas aeruginosa BSI

\begin{tabular}{|c|c|}
\hline Parameter & Patients n (\%) \\
\hline \multicolumn{2}{|l|}{ Basic parameter } \\
\hline Age $>65$ years & $54(48)$ \\
\hline Male sex & $71(63)$ \\
\hline Nosocomial infection & $66(58)$ \\
\hline Fatal outcome & $43(38)$ \\
\hline \multicolumn{2}{|l|}{ Comorbid conditions } \\
\hline Diabetes & $37(33)$ \\
\hline HIV & $1(1)$ \\
\hline Hematological cancer & $36(32)$ \\
\hline Cardiovascular disease & $66(58)$ \\
\hline Pulmonary disease & $11(10)$ \\
\hline Neurologic disease & $24(21)$ \\
\hline Renal disease & $11(10)$ \\
\hline Neutropenia & $38(34)$ \\
\hline \multicolumn{2}{|l|}{ Origin of bacteremia } \\
\hline Unknown (primary BSI) & $50(44)$ \\
\hline Secondary BSI & $55(49)$ \\
\hline Respiratory tract & $21(19)$ \\
\hline Urinary tract & $18(16)$ \\
\hline Intra-Abdominal & $5(4)$ \\
\hline Surgical Site & $1(1)$ \\
\hline Non-surgical site & $10(9)$ \\
\hline VC BSI & $8(7)$ \\
\hline \multicolumn{2}{|l|}{ BSI pathogen } \\
\hline MBL-PA & $18(16)$ \\
\hline 3/4MDR-PA & $27(24)$ \\
\hline MEM-resistant PA & $32(28)$ \\
\hline CAZ-resistant PA & $23(20)$ \\
\hline CIP-resistant PA & $39(35)$ \\
\hline TZP-resistant PA & $34(30)$ \\
\hline
\end{tabular}

BSI, Bloodstream infection; HIV, Human immunodeficiency virus infection; VC BSI, vascular catheter-related blood stream infection; MBL-PA, metallo$\beta$-lactamase producing Pseudomonas aeruginosa; 3/4MDR-PA, 3/4MDRPseudomonas aeruginosa; PA, Pseudomonas aeruginosa; MEM, Meropenem; CAZ, Ceftazidime; CIP, Ciprofloxacin; TZP, Piperacillin-tazobactam.

resistant to meropenem (MEM-resistant PA) were significantly associated with mortality (HR $1.98, P=0.03$, and HR 1.98, $P=0.03$, respectively), this relationship was less conclusive for MBL-PA (HR 1.83, $P=0.1$ ) and ceftazidime resistant $P$. aeruginosa (CAZ-resistant PA, HR $1.88, P=0.06$ ). Ciprofloxacin resistant $P$. aeruginosa (CIP-resistant PA) and piperacillin-tazobactam resistant $P$. aeruginosa (TZP-resistant PA) showed no significant impact on mortality ( $P=0.43$ and 0.28 , respectively). Of note, appropriate empirical treatment turned out not to 
Table 3 Baseline characteristics, comorbidities and treatment parameters of different multidrug-resistant Pseudomonas aeruginosa

\begin{tabular}{|c|c|c|c|c|c|c|}
\hline Parameter & MBL $(n=18)$ & Non-MBL $(n=95)$ & P-value & $3 / 4 M D R(n=27)$ & Non-3/4MDR $(n=86)$ & P-value \\
\hline \multicolumn{7}{|l|}{ Basic parameters } \\
\hline Age, years* & $60(53-64)$ & $67(52-74)$ & 0.16 & $59(48-64)$ & $68.5(54-75)$ & 0.01 \\
\hline Male sex (\%) & $11(61)$ & $60(63)$ & 0.87 & $17(63)$ & $54(63)$ & 0.99 \\
\hline Fatal outcome (\%) & $11(61)$ & $32(34)$ & 0.03 & $17(63)$ & $26(30)$ & 0.002 \\
\hline Length of stay, days* & $23(20-45)$ & $15(8-41)$ & 0.03 & $23(13-45)$ & $14.5(7-41)$ & 0.03 \\
\hline \multicolumn{7}{|l|}{ Comorbid conditions } \\
\hline Immune suppression (\%) & $18(100)$ & $76(80)$ & 0.04 & $27(100)$ & $67(78)$ & 0.006 \\
\hline Neutropenia (\%) & $15(83)$ & $23(24)$ & $<0.001$ & $17(63)$ & $21(24)$ & $<0.001$ \\
\hline Cardiovascular disease (\%) & $7(39)$ & $59(62)$ & 0.07 & $11(41)$ & $55(64)$ & 0.03 \\
\hline Diabetes (\%) & $5(28)$ & $32(34)$ & 0.62 & $7(26)$ & $30(35)$ & 0.39 \\
\hline Pulmonary disease (\%) & $1(6)$ & $10(11)$ & 1 & $1(4)$ & $10(12)$ & 0.46 \\
\hline Neurological disease (\%) & $2(11)$ & $22(23)$ & 0.35 & $6(22)$ & $18(21)$ & 0.89 \\
\hline Renal disease (\%) & $0(0)$ & $11(12)$ & 0.21 & $1(4)$ & $10(12)$ & 0.46 \\
\hline Haematological cancer (\%) & $17(94)$ & $19(20)$ & $<0.001$ & $20(74)$ & $16(19)$ & $<0.001$ \\
\hline Charlson Comorbidity Score* & $2(2-3)$ & $3(1-5)$ & 0.86 & $2(2-3)$ & $3(2-5)$ & 0.27 \\
\hline \multicolumn{7}{|l|}{ Patients clinical record } \\
\hline SAPS $\|^{*}$ & $39.5(36-44)$ & $32(23-41)$ & 0.002 & $37(28-43)$ & $32(24-41)$ & 0.57 \\
\hline AET (\%) & $8(44)$ & $66(70)$ & 0.04 & $11(41)$ & $63(73)$ & 0.002 \\
\hline ADT (\%) & $13(72)$ & $83(87)$ & 0.14 & $19(70)$ & $77(90)$ & 0.03 \\
\hline
\end{tabular}

*Median (interquartile range).

MBL-PA, metallo- $\beta$-lactamase producing Pseudomonas aeruginosa; 3/4MDR-PA, 3/4MDR-Pseudomonas aeruginosa; SAPS II, Simplified Acute Physiology Score II; AET, appropriate empirical treatment; ADT, appropriate definitive treatment.

be a predictor of mortality $(P=0.52)$. Neither MBL-PA nor 3/4MDR-PA or $P$. aeruginosa strains with a resistance to certain antimicrobial agents (MEM, CAZ, CIP, TZP) had an effect on length of stay (LOS) when the entire cohort was investigated. Including only the survivors in the analysis $(n=70)$, a weak association between MBLPA, 3/4MDR-PA and LOS became apparent $(P=0.08$ and $P=0.06$, respectively), indicating an extended LOS for those patients infected with the multidrug-resistant strains (HR 0.49 and 0.55 , respectively).

In multivariate models, the SAPS II score (HR 1.046), cardiovascular disease (HR range 0.44-0.46) and appropriate definitive treatment (HR range 0.25-0.26) were significantly associated with mortality. After adjusting for these confounders, neither MBL-PA nor 3/4MDR-PA remained associated with mortality (Table 5). Also, resistance to either meropenem, ceftazidime, ciprofloxacin or piperacillin-tazobactam on its own was not associated with mortality (data not shown). Furthermore, we did not observe a significant influence of MBL-PA or 3/4MDR-PA on LOS in the fully adjusted models (Table 5). Instead, the presence of concomitant infections was a significant predictor of discharge in all models $(\mathrm{HR}<1)$.

\section{Multi locus sequence typing results}

To investigate whether the results could be influenced by the genetic relatedness of our strains, we performed molecular typing of all 27 isolates exhibiting a multidrugresistant phenotype (3/4MDR). Ten different sequence types were observed (data not shown). Of note, the fourteen IMP-8 producing isolates belonged to the sequence type 308. VIM-2 producing isolates belonged to the sequence types $233(\mathrm{n}=3)$ and $395(\mathrm{n}=1)$. Non-3/4MDRPA $(n=86)$ showed a wide variety of resistance phenotypes and were therefore considered to have a low degree of genetic relatedness.

\section{Discussion}

Despite the extensive spread of MBL-producing organisms, the influence of such pathogens on clinical outcomes has not been comprehensively assessed. The present study showed that MBL-PA BSI results in a higher in-hospital mortality than BSI with Non-MBLPA. These results are in line with earlier reports [14-16]. However, BSI with MBL-PA did not turn out to be an independent predictor of mortality. The higher mortality in the MBL-PA group seemed mainly mediated by the severity of the underlying diseases, as previously reported by 
Table 4 Univariate analysis: Hazard ratios for deaths and discharge (dead or alive) in patients with Pseudomonas aeruginosa BSI

\begin{tabular}{|c|c|c|c|c|c|c|}
\hline Exposure & $\begin{array}{l}\text { Hazard ratio for } \\
\text { deaths }(95 \% \mathrm{Cl})\end{array}$ & P-value & $\begin{array}{l}\text { Hazard ratio for } \\
\text { discharge-time } \\
\text { adjusted }(95 \% \mathrm{Cl})\end{array}$ & P-value & $\begin{array}{c}\text { Hazard ratio for } \\
\text { discharge-time adjusted } \\
(95 \% \mathrm{Cl}) \text { only survivors }(n=70)\end{array}$ & P-value \\
\hline \multicolumn{7}{|l|}{ Basic parameter } \\
\hline Male sex & $1.02(0.55-1.88)$ & 0.95 & $1.14(0.77-1.68)$ & 0.5 & $1.28(0.77-2.12)$ & 0.32 \\
\hline Age, years & $1.0073(0.9889-1.0261)^{*}$ & 0.43 & $1.0164(1.0039-1.0291)^{*}$ & 0.007 & $1.0155(0.9938-1.033)^{*}$ & 0.07 \\
\hline Nosocomial infection & $2.14(1.05-4.27)$ & 0.03 & $0.67(0.42-1.07)$ & 0.1 & $0.52(0.28-0.97)$ & 0.04 \\
\hline \multicolumn{7}{|l|}{ Origin of bacteremia } \\
\hline Unknown (primary BSI) & $1.07(0.57-2)$ & 0.83 & $1.85(1.24-2.75)$ & 0.003 & $2.22(1.32-3.73)$ & 0.003 \\
\hline Secondary BSI & $1.25(0.67-2.31)$ & 0.47 & $0.79(0.53-1.16)$ & 0.24 & $0.76(0.46-1.25)$ & 0.29 \\
\hline VC BSI & $0.22(0.03-1.66)$ & 0.06 & $0.39(0.18-0.85)$ & 0.008 & $0.37(0.15-0.89)$ & 0.01 \\
\hline \multicolumn{7}{|l|}{ Comorbid conditions } \\
\hline Immune suppression & $2.31(0.71-7.55)$ & 0.12 & $0.54(0.31-0.94)$ & 0.04 & $0.5(0.26-0.96)$ & 0.04 \\
\hline Haematological cancer & $1.23(0.67-2.28)$ & 0.5 & $0.61(0.39-0.93)$ & 0.02 & $0.5(0.27-0.91)$ & 0.02 \\
\hline Chemotherapy & $0.83(0.45-1.56)$ & 0.58 & $0.62(0.41-0.94)$ & 0.02 & $0.6(0.35-1.03)$ & 0.06 \\
\hline Cardiovascular disease & $0.65(0.36-1.2)$ & 0.18 & $0.77(0.52-1.14)$ & 0.2 & $0.83(0.49-1.38)$ & 0.48 \\
\hline Diabetes & $1.43(0.77-2.65)$ & 0.25 & $0.78(0.51-1.19)$ & 0.25 & $0.55(0.31-0.99)$ & 0.04 \\
\hline Charlson Comorbidity Score & $0.96(0.83-1.11)^{*}$ & 0.66 & $1(0.92-1.1)^{*}$ & 0.83 & $1.01(0.9-1.13)^{*}$ & 0.77 \\
\hline \multicolumn{7}{|l|}{ Patients clinical record } \\
\hline SAPS $\|$ & $1.0375(1.0184-1.057)^{*}$ & $<0.001$ & $0.9961(0.9828-1.0097)^{*}$ & 0.58 & $0.974(0.9557-0.9926)^{*}$ & 0.005 \\
\hline Neutropenia & $1.41(0.77-2.58)$ & 0.27 & $0.62(0.4-0.94)^{\dagger}$ & 0.02 & $0.43(0.23-0.79)$ & 0.004 \\
\hline Chemotherapy & $0.83(0.45-1.56)$ & 0.58 & $0.62(0.41-0.94)$ & 0.02 & $0.6(0.35-1.03)$ & 0.06 \\
\hline Steroids & $1.82(0.86-3.84)$ & 0.1 & $0.46(0.3-0.7)$ & $<0.001$ & $0.3(0.17-0.54)$ & $<0.001$ \\
\hline Concomitant infections & $1.22(0.56-2.71)$ & 0.59 & $0.27(0.16-0.44)$ & $<0.001$ & $0.23(0.11-0.46)$ & $<0.001$ \\
\hline Recent surgery & $0.65(0.34-1.22)$ & 0.18 & $0.54(0.35-0.81)$ & 0.003 & $0.47(0.27-0.82)$ & 0.007 \\
\hline AET & $0.81(0.44-1.5)$ & 0.52 & $0.89(0.59-1.34)$ & 0.6 & $0.9(0.53-1.51)$ & 0.7 \\
\hline ADT & $0.33(0.16-0.68)$ & 0.007 & $0.44(0.25-0.77)$ & 0.008 & $0.4(0.17-0.92)$ & 0.052 \\
\hline \multicolumn{7}{|l|}{ Pathogen exposure } \\
\hline MBL-PA & $1.83(0.92-3.64)$ & 0.1 & $0.75(0.44-1.27)$ & 0.27 & $0.49(0.21-1.17)$ & 0.08 \\
\hline 3/4MDR-PA & $1.98(1.07-3.67)$ & 0.03 & $0.76(0.49-1.19)$ & 0.23 & $0.55(0.25-1.07)$ & 0.06 \\
\hline MEM-resistant PA & $1.98(1.08-3.62)$ & 0.03 & $0.85(0.55-1.3)$ & 0.45 & $0.64(0.33-1.23)$ & 0.17 \\
\hline CAZ-resistant PA & $1.88(0.99-3.57)$ & 0.06 & $0.72(0.45-1.16)$ & 0.17 & $0.53(0.25-1.15)$ & 0.09 \\
\hline CIP-resistant PA & $1.27(0.69-2.34)$ & 0.43 & $0.93(0.62-1.39)$ & 0.73 & $0.78(0.45-1.34)$ & 0.38 \\
\hline TZP-resistant PA & $1.41(0.76-2.61)$ & 0.28 & $0.78(0.52-1.19)$ & 0.26 & $0.68(0.37-1.23)$ & 0.2 \\
\hline
\end{tabular}

${ }^{\dagger}$ Proportional hazard assumption not fulfilled for this parameter.

*Per 1 unit increase.

95\% Cl, 95\% Confidence interval; BSI, blood stream infection; VC BSI, vascular catheter-related blood stream infection; SAPS II, Simplified Acute Physiology Score; AET, appropriate empirical treatment; ADT, appropriate definitive treatment; MBL-PA, metallo- $\beta$-lactamase producing Pseudomonas aeruginosa; $3 / 4 \mathrm{MDR}-\mathrm{PA}$, 3/4MDR-Pseudomonas aeruginosa; PA, Pseudomonas aeruginosa; MEM, Meropenem; CAZ, Ceftazidime; CIP, Ciprofloxacin; TZP, Piperacillin-tazobactam.

Zavascki and colleagues in patients with various nosocomial infections [16]. While the administration of appropriate definitive treatment had independently a strong protective effect (HR range $0.25-0.26$ ), there was only a weak tendency that patients with MBL-PA BSI were less likely to have received sufficient therapy. After the emergence of the first MBL-PA isolates, colistin (colistimethate sodium) was added to the empirical therapeutic regime when a BSI with $P$. aeruginosa was suspected. MBL-PA isolates were resistant to all antimicrobial agents except for colistin. In fact, colistin was intravenously administered to 13 of 18 patients with MBL-PA BSI (72\%), and these patients were considered to have received appropriate therapy. It can be speculated that colistin has improved the outcome of these patients in our setting. This hypothesis is supported by the fact that the treatment 
Table 5 Multivariate analysis: Hazard ratios for deaths and discharge (dead or alive)

\begin{tabular}{|c|c|c|c|c|c|c|}
\hline Parameter & $\begin{array}{l}\text { Hazard ratio for } \\
\text { deaths }(95 \% \mathrm{Cl})\end{array}$ & P-value & $\begin{array}{l}\text { Hazard ratio for } \\
\text { discharge-fully } \\
\text { adjusted ( } 95 \% \mathrm{Cl})\end{array}$ & P-value & $\begin{array}{c}\text { Hazard ratio for } \\
\text { discharge-fully adjusted } \\
(95 \% \mathrm{Cl}) \text { only survivors }(n=70)\end{array}$ & P-value \\
\hline$\overline{M B L-P A}$ & $0.98(0.45-2.1)^{a}$ & 0.97 & $1.28(0.63-2.58)^{c}$ & 0.49 & $1.53(0.53-4.45)^{\mathrm{e}}$ & 0.44 \\
\hline 3/4MDR-PA & $1.37(0.68-2.72)^{b}$ & 0.37 & $1.24(0.69-2.21)^{d}$ & 0.46 & $0.75(0.31-1.83)^{f}$ & 0.53 \\
\hline
\end{tabular}

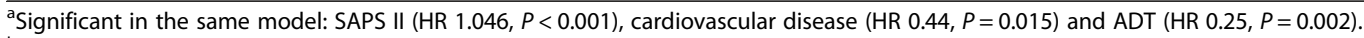

${ }^{b}$ Significant in the same model: SAPS II (HR 1.046, $P<0.001$ ), cardiovascular disease (HR $\left.0.46, P=0.025\right)$ and ADT (HR 0.26, $P=0.003$ ).

'Significant in the same model: Concomitant infection (HR 0.3, $P<0.001)$, ADT (HR 0.39, $P=0.004)$.

dSignificant in the same model: Concomitant infection (HR 0.27, $P<0.001$ ), ADT (HR 0.4, $P=0.007$ ).

eSignificant in the same model: Concomitant infection (HR 0.32, $P=0.002$ ), vascular catheter-related BSI (HR 0.41, $P=0.04)$, nosocomial infection (HR 0.44, $P=0.04$ )

fSignificant in the same model: Concomitant infection (HR 0.32, $P=0.002)$, neutropenia (HR 0.39, $P=0.006)$, nosocomial infection (HR 0.37, $P=0.004)$, ADT (HR 0.25, $P=0.008)$.

95\% Cl, 95\% Confidence interval; MBL-PA, metallo- $\beta$-lactamase producing Pseudomonas aeruginosa; 3/4MDR-PA, 3/4MDR-Pseudomonas aeruginosa; SAPS II, Simplified Acute Physiology Score; ADT, appropriate definitive treatment.

variable for definitive therapy proved to be strongly protective when regarding colistin as appropriate treatment in the multivariate models. However, our study was not designed to resolve this issue. And despite the growing evidence for the therapeutic benefit of intravenous colistin in adult patients infected with multidrug-resistant $P$. aeruginosa [30-32], prospective and randomized control trials are required to prove its efficacy and safety.

Different phenotypes of resistance of $P$. aeruginosa in BSI did not prove to be independent predictors of mortality, either. In the case of 3/4MDR-PA, the observed difference in mortality compared to Non-3/4MDR-PA (63\% vs $30 \%, P=0.002$, Table 3 ) appeared to be due to the lower chance of patients with 3/4MDR-PA BSI to have received appropriate definitive treatment. This emphasizes the importance of reevaluating the initial therapeutic regimes in a hospital setting with a relevant incidence of multidrug-resistant $P$. aeruginosa. It also stresses the importance of susceptibility testing results from the associated microbiology laboratories and the need to effectively and appropriately adjust empirical treatments where necessary. Whether multidrug-resistant phenotypes of $P$. aeruginosa have an intrinsic influence on the risk of a lethal outcome is still controversial. While some studies revealed infection with multidrug-resistant $P$. aeruginosa to be an independent predictor of mortality $[3,33,34]$ others have not observed such a connection $[35,36]$. The reasons for this heterogeneity are unknown. However, one possibility is that not all of these studies performed molecular genotyping, and their results could have been influenced by the presence of just a few dominant strains, possibly in possession of virulence factors that could have contributed to worse clinical outcomes [37]. These or similar circumstances may have led to confounding effects. Although we did not perform molecular typing for all isolates, the identification of 10 different MLST sequence types among the multidrug-resistant phenotypes (3/4MDR-PA) in our study makes such a confounding less likely, with the exception of the 18 MBL-PA isolates which belong to three different strains. These strains circulated in only a few wards at the tertiary teaching hospital, suggesting that transmission within the hospital is still a major factor for the spread of MBL-PA. Another reason for our observations might be the limitation in study power. Since we needed to get by with less than 10 outcome events per variable in our multivariate models, we may have been incapable of detecting a minor effect of MBL producers or resistant phenotypes on mortality. Nevertheless, a relevant influence on mortality is unlikely to have been overlooked. Larger-scaled, multicenter studies are still required to investigate this issue and to generalize results to other settings with confidence.

The absence of a significant relation between an appropriate empirical treatment (within 24 hours) and mortality is another interesting finding. Lodise and colleagues noted that the risk of a fatal outcome rises in patients with $P$. aeruginosa BSI once the delay in receiving appropriate treatment exceeded 52 hours [36]. However, their study did not provide information about the probable origin of infection. This might be an important point since interventions like abolition of urinary obstructions and removal of catheters are the mainstay of therapy in blood stream infections with the urinary tract or a vascular catheter as probable origins of infection, leaving prompt antimicrobial treatment no more than a secondary role in the clinical management. This subpopulation of our patients (origin of infection: $16 \%$ urinary tract and $7 \%$ vascular catheterrelated, Table 1) may have influenced the results in the observed direction. It must be noted that this proportion may be an underestimation due to our study's limitation as a retrospective investigation. However, we excluded patients among whom vital clinical information was missing (4 of 120 patients) and are thus confident that the data accurately represents the real situation to a high degree.

In addition, we investigated whether MBL production or resistance phenotypes were associated with the length of stay. BSI with a MBL producer or resistant phenotype did not prolong the LOS, while the presence of concomitant infections besides a PA-BSI did so $(\mathrm{HR}<1$, all models). In fully adjusted models wherein only survivors 
were included, nosocomial infections were also significantly associated with a prolonged stay. These results indicate that any concomitant infection should be avoided to reduce additional morbidity and hospital costs, leading to the demand for strict routine infection control measures.

\section{Conclusions}

The administration of appropriate definitive treatment in patients with PA-BSI, even when delayed, remains the backbone for improving clinical outcomes and should be combined with routine infection control measures to further reduce morbidity and costs. The pattern of resistance within a hospital should be continuously monitored for a rapid detection of changes and consecutive adjustment of empirical treatment regimes.

\section{Competing interests}

Our study was supported in part by the German Centre of Infection Research (DZIF). The funder had no role in study design, data collection and analysis, decision to publish, or preparation of the manuscript. The authors declare that they have no competing interests.

\section{Authors' contributions}

MW led the investigation, including design of the cohort study. IK, MM, KS, FH, WV, IF and UM collected clinical and epidemiological data. Laboratory analysis was conducted by SP, MM and IBA. MW and SP analyzed the data and wrote the manuscript. All authors read, commented on and approved the final manuscript.

\section{Acknowledgements}

We give thanks to the directors, physicians, laboratory and nursing staff of the medical wards in all participating hospitals. We would further like to thank Kerstin Fischer, Nadine Hoffmann and Nenad Katava for their support in antimicrobial susceptibility testing and the general assistance. Finally, we would like to extend our gratitude to the staff of the National Reference Laboratory for multidrug-resistant gram-negative bacteria (Bochum, Germany) for additional molecular characterization of five isolates.

\section{Author details}

'Institute of Medical Microbiology and Hygiene, University of Tübingen, Elfriede-Aulhorn-Str. 6, 72076 Tübingen, Germany. ${ }^{2}$ Medical Center, Department of Hematology, Oncology, Immunology, Rheumatology \& Pulmonology, University of Tübingen, Tübingen, Germany. ${ }^{3}$ BG Trauma Center, University of Tübingen, Tübingen, Germany. ${ }^{4}$ Clinic for General, Visceral and Vascular Surgery, Zollernalb Hospital, Albstadt, Germany.

Received: 24 July 2013 Accepted: 29 October 2013

Published: 1 November 2013

\section{References}

1. Gould IM, Wise R: Pseudomonas aeruginosa: clinical manifestations and management. Lancet 1985, 2(8466):1224-1227.

2. Kang Cl, Kim SH, Kim HB, Park SW, Choe YJ, Oh MD, Kim EC, Choe KW: Pseudomonas aeruginosa bacteremia: risk factors for mortality and influence of delayed receipt of effective antimicrobial therapy on clinical outcome. Clin Infect Dis 2003, 37(6):745-751.

3. Tumbarello M, Repetto E, Trecarichi EM, Bernardini C, De Pascale G, Parisini A, Rossi M, Molinari MP, Spanu T, Viscoli C, et al: Multidrug-resistant Pseudomonas aeruginosa bloodstream infections: risk factors and mortality. Epidemiol Infect 2011, 139(11):1740-1749.

4. Goel N, Wattal C, Oberoi JK, Raveendran R, Datta S, Prasad KJ: Trend analysis of antimicrobial consumption and development of resistance in non-fermenters in a tertiary care hospital in Delhi, India. J Antimicrob Chemother 2011, 66(7):1625-1630.
5. Croughs PD, Li B, Hoogkamp-Korstanje JA, Stobberingh E: Thirteen years of antibiotic susceptibility surveillance of Pseudomonas aeruginosa from intensive care units and urology services in the Netherlands. Eur I Clin Microbiol Infect Dis 2013, 32(2):283-288.

6. Lautenbach E, Synnestvedt M, Weiner MG, Bilker WB, Vo L, Schein J, Kim M: Imipenem resistance in Pseudomonas aeruginosa: emergence, epidemiology, and impact on clinical and economic outcomes. Infect Control Hosp Epidemiol 2010, 31(1):47-53.

7. Willmann M, Marschal M, Holzl F, Schroppel K, Autenrieth IB, Peter S: Time series analysis as a tool to predict the impact of antimicrobial restriction in antibiotic stewardship programs using the example of multidrugresistant Pseudomonas aeruginosa. Antimicrob Agents Chemother 2013, 57(4):1797-1803.

8. Valenza G, Joseph B, Elias J, Claus H, Oesterlein A, Engelhardt K, Turnwald D, Frosch M, Abele-Horn M, Schoen C: First survey of metallo-betalactamases in clinical isolates of Pseudomonas aeruginosa in a German university hospital. Antimicrob Agents Chemother 2010, 54(8):3493-3497.

9. Cornaglia G, Giamarellou H, Rossolini GM: Metallo-beta-lactamases: a last frontier for beta-lactams? Lancet Infect Dis 2011, 11(5):381-393.

10. Riccio ML, Pallecchi L, Fontana R, Rossolini GM: In70 of plasmid pAX22, a bla(VIM-1)-containing integron carrying a new aminoglycoside phosphotransferase gene cassette. Antimicrob Agents Chemother 2001, 45(4):1249-1253.

11. Scoulica EV, Neonakis IK, Gikas Al, Tselentis YJ: Spread of bla(VIM-1)producing $\mathrm{E}$. coli in a university hospital in Greece: genetic analysis of the integron carrying the bla(VIM-1) metallo-beta-lactamase gene. Diagn Microbiol Infect Dis 2004, 48(3):167-172.

12. Bush K: New beta-lactamases in gram-negative bacteria: diversity and impact on the selection of antimicrobial therapy. Clin Infect Dis 2001, 32(7):1085-1089.

13. Giske CG, Libisch B, Colinon C, Scoulica E, Pagani L, Fuzi M, Kronvall G, Rossolini GM: Establishing clonal relationships between VIM-1-like metallo-beta-lactamase-producing Pseudomonas aeruginosa strains from four European countries by multilocus sequence typing. J Clin Microbiol 2006, 44(12):4309-4315.

14. Hirakata Y, Yamaguchi T, Nakano M, Izumikawa K, Mine M, Aoki S, Kondoh A, Matsuda J, Hirayama M, Yanagihara K, et al: Clinical and bacteriological characteristics of IMP-type metallo-beta-lactamase-producing Pseudomonas aeruginosa. Clin Infect Dis 2003, 37(1):26-32.

15. Laupland KB, Parkins MD, Church DL, Gregson DB, Louie TJ, Conly JM, Elsayed S, Pitout JD: Population-based epidemiological study of infections caused by carbapenem-resistant Pseudomonas aeruginosa in the Calgary health region: importance of metallo-beta-lactamase (MBL)producing strains. J Infect Dis 2005, 192(9):1606-1612.

16. Zavascki AP, Barth AL, Goncalves AL, Moro AL, Fernandes JF, Martins AF, Ramos F, Goldani LZ: The influence of metallo-beta-lactamase production on mortality in nosocomial Pseudomonas aeruginosa infections. J Antimicrob Chemother 2006, 58(2):387-392.

17. Vandenbroucke JP, Von Elm E, Altman DG, Gotzsche PC, Mulrow CD, Pocock SJ, Poole C, Schlesselman JJ, Egger M: Strengthening the reporting of observational studies in epidemiology (STROBE): explanation and elaboration. PLoS Med 2007, 4(10):e297.

18. Definition of multidrug-resistance to antimicrobial agents in Gram-negative rods with regards to preventative infection control measures. http://www.rki. de/DE/Content/Infekt/EpidBull/Archiv/2011/Ausgaben/36_11.pdf?_blob= publicationFile.

19. Calandra T, Cohen J: The international sepsis forum consensus conference on definitions of infection in the intensive care unit. Crit Care Med 2005, 33(7):1538-1548.

20. Charlson ME, Pompei P, Ales KL, Mackenzie CR: A new method of classifying prognostic comorbidity in longitudinal studies: development and validation. J Chronic Dis 1987, 40(5):373-383.

21. Le Gall JR, Lemeshow S, Saulnier F: A new simplified acute physiology score (SAPS II) based on a European/North American multicenter study. JAMA 1993, 270(24):2957-2963.

22. Breakpoint tables for interpretation of MICs and zone diameters. http://www. eucast.org/fileadmin/src/media/PDFs/EUCAST_files/Breakpoint_tables/ Breakpoint_table_v_2.0_120221.pdf.

23. Leclercq R, Canton R, Brown DF, Giske CG, Heisig P, Macgowan AP, Mouton JW, Nordmann P, Rodloff AC, Rossolini GM, et al: EUCAST expert rules in antimicrobial susceptibility testing. Clin Microbiol Infect 2011, 19(2):141-160. 
24. EUCAST Disk Diffusion Method for Antimicrobial Susceptibility Testing. www. eucast.org.

25. CLSI: Performance standards for antimicobial susceptibility testing. 32nd edition. Wayne, PA: Clinical and Laboratory Standards Institute; 2012.

26. Pitout JD, Gregson DB, Poirel L, McClure JA, Le P, Church DL: Detection of Pseudomonas aeruginosa producing metallo-beta-lactamases in a large centralized laboratory. J Clin Microbiol 2005, 43(7):3129-3135.

27. Lee MF, Peng CF, Hsu HJ, Chen YH: Molecular characterisation of the metallo-beta-lactamase genes in imipenem-resistant Gram-negative bacteria from a university hospital in southern Taiwan. Int I Antimicrob Agents 2008, 32(6):475-480.

28. Lambert ML, Suetens C, Savey A, Palomar M, Hiesmayr M, Morales I, Agodi A, Frank U, Mertens K, Schumacher M, et al: Clinical outcomes of healthcare-associated infections and antimicrobial resistance in patients admitted to European intensive-care units: a cohort study. Lancet Infect Dis 2011, 11(1):30-38.

29. Abbo A, Carmeli Y, Navon-Venezia S, Siegman-Igra Y, Schwaber MJ: Impact of multi-drug-resistant Acinetobacter baumannii on clinical outcomes. Eur J Clin Microbiol Infect Dis 2007, 26(11):793-800.

30. Durakovic N, Radojcic V, Boban A, Mrsic M, Sertic D, Serventi-Seiwerth R, Nemet D, Labar B: Efficacy and safety of colistin in the treatment of infections caused by multidrug-resistant Pseudomonas aeruginosa in patients with hematologic malignancy: a matched pair analysis. Intern Med 2011, 50(9):1009-1013.

31. Hachem RY, Chemaly RF, Ahmar CA, Jiang Y, Boktour MR, Rjaili GA, Bodey GP, Raad II: Colistin is effective in treatment of infections caused by multidrug-resistant Pseudomonas aeruginosa in cancer patients. Antimicrob Agents Chemother 2007, 51(6):1905-1911.

32. Montero M, Horcajada JP, Sorli L, Alvarez-Lerma F, Grau S, Riu M, Sala M, Knobel H: Effectiveness and safety of colistin for the treatment of multidrug-resistant Pseudomonas aeruginosa infections. Infection 2009, 37(5):461-465.

33. Hirsch EB, Cottreau JM, Chang KT, Caeiro JP, Johnson ML, Tam VH: A model to predict mortality following Pseudomonas aeruginosa bacteremia. Diagn Microbiol Infect Dis 2012, 72(1):97-102.

34. Aloush V, Navon-Venezia S, Seigman-Igra Y, Cabili S, Carmeli Y: Multidrugresistant Pseudomonas aeruginosa: risk factors and clinical impact. Antimicrob Agents Chemother 2006, 50(1):43-48.

35. Morata L, Cobos-Triqueros N, Martinez JA, Soriano A, Almela M, Marco F, Sterzik H, Nunez R, Hernandez C, Mensa J: Influence of multidrug resistance and appropriate empirical therapy on the 30-day mortality rate of Pseudomonas aeruginosa bacteremia. Antimicrob Agents Chemother 2012, 56(9):4833-4837.

36. Lodise TP Jr, Patel N, Kwa A, Graves J, Furuno JP, Graffunder E, Lomaestro B, McGregor JC: Predictors of 30-day mortality among patients with Pseudomonas aeruginosa bloodstream infections: impact of delayed appropriate antibiotic selection. Antimicrob Agents Chemother 2007, 51(10):3510-3515.

37. El-Solh AA, Hattemer A, Hauser AR, Alhajhusain A, Vora H: Clinical outcomes of type III Pseudomonas aeruginosa bacteremia. Crit Care Med 2012, 40(4):1157-1163

doi:10.1186/1471-2334-13-515

Cite this article as: Willmann et al: Effect of metallo- $\beta$-lactamase production and multidrug resistance on clinical outcomes in patients with Pseudomonas aeruginosa bloodstream infection: a retrospective cohort study. BMC Infectious Diseases 2013 13:515.

\section{Submit your next manuscript to BioMed Central and take full advantage of:}

- Convenient online submission

- Thorough peer review

- No space constraints or color figure charges

- Immediate publication on acceptance

- Inclusion in PubMed, CAS, Scopus and Google Scholar

- Research which is freely available for redistribution

Submit your manuscript at www.biomedcentral.com/submit 